Check for updates

Cite this: Chem. Commun., 2018, 54,8530

Received 29th May 2018, Accepted 29th June 2018

DOI: $10.1039 / \mathrm{c} 8 \mathrm{cc} 04267 \mathrm{e}$

rsc.li/chemcomm

\section{Multiplex imaging of live breast cancer tumour models through tissue using handheld surface enhanced spatially offset resonance Raman spectroscopy (SESORRS) $\dagger$}

\author{
Fay Nicolson, (D) ${ }^{a}$ Lauren E. Jamieson, (D) a Samuel Mabbott, (D) ${ }^{a}$ \\ Konstantinos Plakas, (DD ${ }^{b}$ Neil C. Shand, (D) Michael R. Detty, (D) ${ }^{\mathrm{b}}$ \\ Duncan Graham (iD ${ }^{a}$ and Karen Faulds (iD *a
}

Through utilizing the depth penetration capabilities of SESORS, multiplexed imaging and classification of three singleplex nanotags and a triplex of nanotags within breast cancer tumour models is reported for the first time through depths of $10 \mathrm{~mm}$ using a handheld SORS instrument.

With the number of new cases of cancer expected to rise by approximately $70 \%$ in the next two decades, ${ }^{1}$ non-invasive tumour detection is of profound importance. Surface enhanced Raman scattering (SERS) has emerged as a promising tool for biomedical imaging. ${ }^{2,3}$ SERS has been deployed in numerous biomedical applications including the detection of cancer in vivo., Surface enhanced resonance Raman scattering (SERRS) utilises a Raman reporter molecule with an electronic transition close to the laser frequency of the Raman instrument, thus generating further enhancement in Raman signal. SERRS holds promise over fluorescence based methods, particularly in multiplexing applications due to the sharp fingerprint spectrum obtained, allowing multiple analyte molecules to be detected simultaneously without the need for separation or multiple excitation wavelengths. ${ }^{6}$ There is great interest in the ability to simultaneously detect multiple biomarkers in a sensitive and non-destructive manner in disease diagnostics. Using confocal techniques, the simultaneous detection of multiple SERS nanotags, ${ }^{7}$ and three breast cancer biomarkers has been reported in vivo. ${ }^{3}$ Here the ability to not only detect but also classify multiple SERRS nanotags taken up into ex vivo tumour models buried at tissue depths of $10 \mathrm{~mm}$ is demonstrated using the technique of surface enhanced spatially offset resonance Raman spectroscopy (SESORRS) for the first time.

\footnotetext{
${ }^{a}$ Department of Pure and Applied Chemistry, Technology and Innovation Centre, University of Strathclyde, 99 George Street, Glasgow G1 1RD, UK. E-mail: karen.faulds@strath.ac.uk

${ }^{b}$ Department of Chemistry, University at Buffalo, The State University of New York, New York 14260, USA

${ }^{c}$ DSTL, Porton Down, Salisbury, SP4 OJQ, UK

$\dagger$ Electronic supplementary information (ESI) available. See DOI: 10.1039/ c8cc04267e
}

Spatially offset Raman spectroscopy (SORS) is an emerging spectroscopic technique useful for the non-invasive analysis of turbid media. Unlike confocal techniques, SORS is better equipped to probe through depth ${ }^{8}$ and uses a spatial offset between the point of laser excitation and point of collection to obtain Raman signal from the deeper layers within a sample. SORS has been deployed in numerous security ${ }^{9,10}$ and biomedical applications including the analysis of bone ${ }^{11}$ and in the identification of breast cancer calcifications. ${ }^{12}$ Surface enhanced spatially offset Raman spectroscopy (SESORS) combines the depth penetration benefits of SORS with the signal enhancing capabilities of SERS to achieve greater sample interrogation at significant depth. ${ }^{8}$ Using a backscattering configuration, SESORS has been used in glucose sensing, ${ }^{13}$ the detection of neurotransmitters ${ }^{14}$ and in the tracking of nanotags through $8 \mathrm{~mm}$ of bone. ${ }^{15}$ Using a transmission approach, Stone et al. detected signal from nanotags through up to $50 \mathrm{~mm}$ of tissue. ${ }^{8,16}$

To the best of our knowledge, the only report on the use of SESORS for multiplex imaging was in a previous collaboration with Stone $e t$ al., where the ability to detect Raman signal from four SERS nanotags through depths of $20 \mathrm{~mm}$ was reported. ${ }^{8}$ The individual nanotags were each injected into four corners of a tissue section. Since the spectral fingerprint of the four nanotags was significantly different for each Raman reporter, it was possible to assign a specific peak in each of the four spectra to enable a spatial multiplex detection using SESORS. However, this approach utilised a benchtop instrument with transmission geometry which could be challenging for some biomedical applications where it may not be possible to collect Raman scattering in a transmission mode.

Using multicellular tumour spheroids (MTS) as ex vivo cancer models, the novel technique of SESORRS has been reported. SESORRS couples the signal enhancing capabilities of SERRS with SORS, to yield enhanced signal at even greater depth. ${ }^{17}$ By tuning the absorbance wavelength of chalcogenpyrylium dyes into the near-infrared (NIR) ${ }^{18}$ Raman reporters that are in resonance with the laser excitation wavelength of $830 \mathrm{~nm}$ can 
be used to generate greater enhancement in Raman signal. As such, the successful detection of single SERRS active nanotags taken up into MTS breast cancer tumour models has been demonstrated through depths of $15 \mathrm{~mm}$ using handheld SESORRS. ${ }^{18}$ Now, using the same ex vivo breast cancer models, the development of a multiplex imaging system capable of detecting a triplex of three SERRS-active NPs present in MTS is reported and the promise of handheld SESORRS with backscattering optics for multiplex applications is shown. Not only does this work highlight the ability to detect multiple analytes at depth, it is the first demonstration of the use of chemometrics to classify a triplex of nanotags located at depths using the SESORRS technique in a 3D tumour model. Furthermore, in comparison to a previous report, ${ }^{8}$ it represents the ability to carry out multiplex detection using SESORRS in a clinically relevant scenario.

To develop a multiplexed imaging system at depth, gold NPs were synthesised according to previously reported methods. ${ }^{18}$ The resulting particles had an average diameter of $78 \mathrm{~nm}$ and were functionalised separately with three different chalcogenpyrylium resonant Raman reporters. These nanotags were then incubated with MCF7 cells, enabling delivery into live breast cancer tumour models to demonstrate the ability to carry out multiplexed detection at depths of $10 \mathrm{~mm}$ in tissue using SESORRS. The chalcogenpyrylium dyes were chosen specifically due to their resonance properties. Previous reports have shown that by exploiting the resonance effect, superior depth penetration can be achieved. ${ }^{17}$ The absorbance wavelength of the highly Raman active molecules is tuned by controlling the number of $\mathrm{sp}^{2}$ carbons in the aliphatic back bone and the chalcogen atoms in the ring system. ${ }^{18}$ The chemical structure of the three Raman reporters used in this work are shown in Fig. 1(a-c). Each reporter is named on their absorbance properties, i.e. dye 810 has an absorbance maxima at $810 \mathrm{~nm}$. The SERRS spectra for each of the three dyes is also shown in Fig. $1 d$.

MTS were used as a 3D breast cancer tumour model to demonstrate the clinical significance of SESORRS for in vivo multiplexing applications. MCF7 human breast cancer cells were incubated overnight with $1 \mathrm{~mL}$ of each of the three nanotag solutions containing dye 810,813 or 823 . MCF7 cells were also incubated with a $1 \mathrm{~mL}$ triplex solution containing $33 \%$ of each of the three SERRS nanotags (13.7 pM of AuNPs), i.e. the final number of NPs remained constant. Incubation resulted in the uptake and accumulation of the nanotags within the cancer cells. MTS were then grown from a suspension of these cells over a period of 7 days to a size $<1 \mathrm{~mm}$. Previous reports show that NPs are homogenously dispersed throughout the MTS. ${ }^{19}$ The dyes did not cause cell death since the cells divided and replicated in order to form the MTS models.

The experimental set up is described in Fig. S1 (ESI $\dagger$ ). Approximately 10 MTS containing either a single SERRS nanotag, i.e. dye 823 , dye 813 or dye 810 , or a triplex of all three nanotags, were transferred to a section of porcine tissue. For example, 10 MTS containing dye 823 were transferred to a single tissue section. Another section of tissue with a $10 \mathrm{~mm}$ thickness was then placed on top of the layer containing the MTS. The same

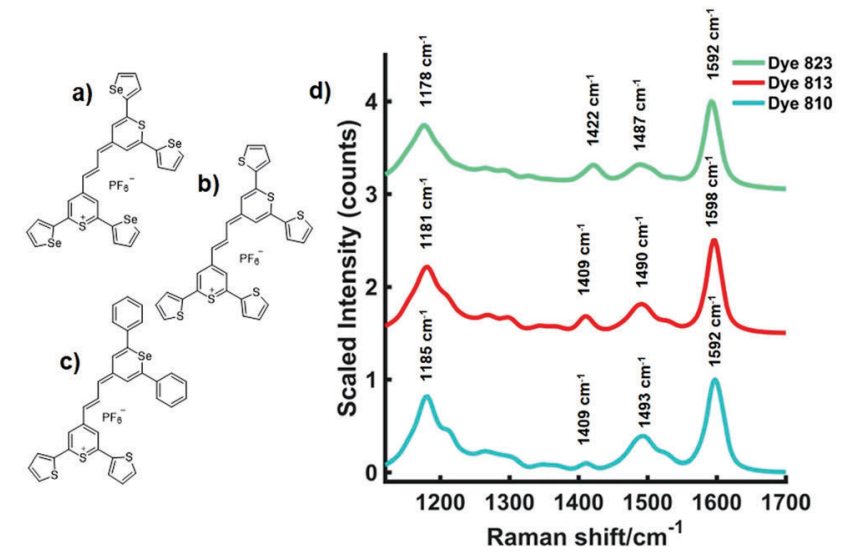

Fig. 1 Chemical structure of dye 823 (a), dye 813 (b) and dye 810 (c). Dyes were named according to the resonances, i.e. dye 823 has an absorbance maximum at $823 \mathrm{~nm}$. (d) SERRS spectra of dyes 823,813 and 810 . All measurements were carried out using a 2 s integration time, 5 accumulations, $830 \mathrm{~nm}$ laser excitation wavelength.

process was applied for the remaining MTS containing the singleplex or triplex nanotags. All MTS models were obscured by $10 \mathrm{~mm}$ of tissue. A translational $x-y$ stage with a range of $2.54 \mathrm{~cm}$ was used to manoeuver the tissue samples in steps of $3 \mathrm{~mm}$ to create an image of $8 \times 8$ pixels. The tissue system was brought into contact with the nose cone, and the $z$-value remained fixed, i.e. the ability to probe through a depth of $10 \mathrm{~mm}$ in the $z$-plane was achieved by using an $8 \mathrm{~mm}$ spatial offset. Thus, by utilising the SESORRS technique, it was possible to detect each of the four individual MTS models through a $10 \mathrm{~mm}$ of tissue barrier.

Using spectra collected at an $8 \mathrm{~mm}$ offset, false colour 2D SESORRS heat maps of the peak intensity at $1178 \mathrm{~cm}^{-1}$ (dye 823) $1181 \mathrm{~cm}^{-1}$ (dye 813), $1185 \mathrm{~cm}^{-1}$ (dye 810 ) and $1181 \mathrm{~cm}^{-1}$ (triplex) were constructed, Fig. 2. The four peaks were chosen to show the position of the MTS through $10 \mathrm{~mm}$ of tissue and correspond to the uptake of both the single nanotags and triplex of nanotags into MTS, rather than the specific contribution of each dye to the acquired spectrum. The false colour images show clear discrimination between areas where the MTS models containing the SERRS-active nanotags were present and where they were not. As such, the areas of maximum intensity in each of the four heat maps correspond to the regions in which the MTS models were positioned (Fig. 2a-d). This can be used as a means to determine if detection of the MTS models has taken place since a SERRS response is observed from the uptake of nanotags into the MTS. The offset spectra collected at the point of maximum intensity, (Fig. 2a-d, purple spectra) shows clear detection of MTS containing either single nanotags or a triplex of the three nanotags. The spectra collected at the point of minimum intensity (Fig. 2a-d, green) corresponds to the tissue. Thus, by comparing the spectra collected at the point of maximum and minimum intensity through the same barrier thickness of $10 \mathrm{~mm}$ of tissue, successful detection of MTS containing either single or a triplex of varying SERRS nanotags is demonstrated.

The focus of the work presented here is to demonstrate the ability to distinguish between multiple nanotags and to 

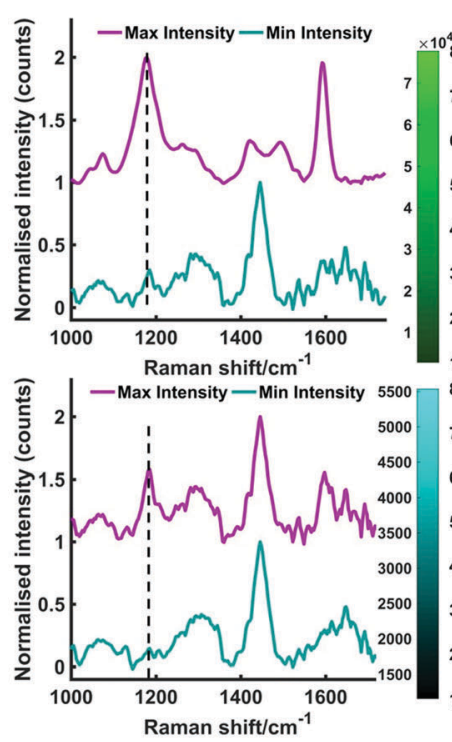

a) Dye823
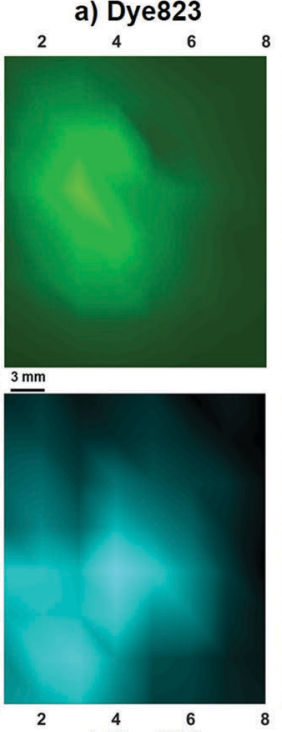

c) $\stackrel{4}{\text { Dye } 810}$ b) Dye813
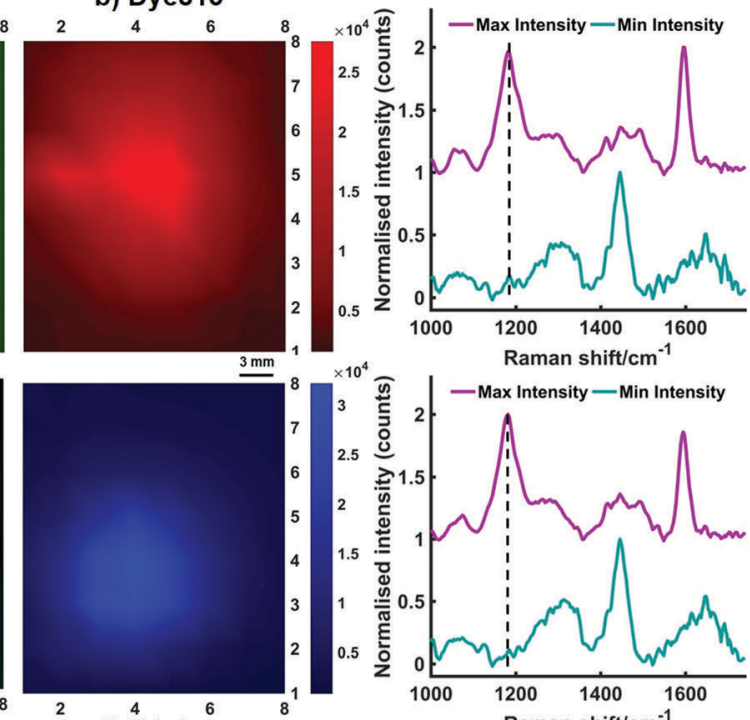

d) Triplex

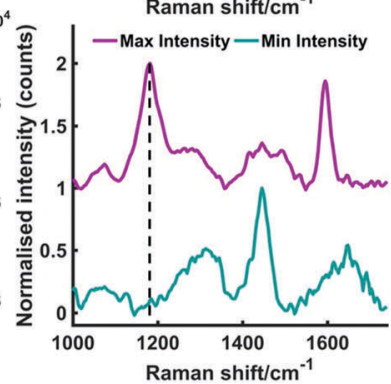

Fig. 2 SESORRS false colour 2D heat maps of the peak intensity at (a) $1178 \mathrm{~cm}^{-1}$ (dye 823), (b) $1181 \mathrm{~cm}^{-1}$ (dye 813 ), (c) $1185 \mathrm{~cm}^{-1}$ (dye 810 ) and (d) $1181 \mathrm{~cm}^{-1}$ (triplex). Measurements were carried out using an $x y$ translational stage in step sizes of $3 \mathrm{~mm}$ to create an image of $8 \times 8$ pixels. 2D heat maps were generated and show the tracking of each of the four MTS models through $10 \mathrm{~mm}$ of tissue. Clear discrimination is seen between spectra collected at the point of maximum intensity where the nanotags were spotted and that collected where the nanotags were not present. The corresponding maximum and minimum collected $8 \mathrm{~mm}$ offset spectra also confirm the presence of the nanotags in regions where the MTS were spotted (a-d). All measurements were carried out using a $2 \mathrm{~s}$ integration time, 5 accumulations, $830 \mathrm{~nm}$ laser excitation wavelength.

specifically classify a single nanotag and a triplex of the three nanotags through depth using SESORRS. Since strong spectral similarity exists between the spectra from the three individual SERRS nanotags and the triplex, principal component analysis (PCA) was applied to analyse the data from the SESORRS multiplex image. ${ }^{20}$ Offset spectra were acquired at the point of maximum intensity through $10 \mathrm{~mm}$ of tissue, i.e. through $10 \mathrm{~mm}$ of tissue in the region where the MTS models were present. Sets of spectra were acquired for both the single nanotags taken up into the MTS models and the MTS models containing the triplex. Reference spectra were also obtained from aqueous solutions of the nanotags containing each of the three single nanotags and a mixture composed of equal amounts of each solution (triplex) which were deposited into a quartz microcuvette (path length $1 \mathrm{~mm}$, chamber volume $350 \mu \mathrm{L}$ ) and placed behind $10 \mathrm{~mm}$ of porcine tissue. The final concentration of dye in both the single and triplex solutions was $300 \mathrm{nM}$. The experimental set up is described in the ESI $\dagger$ and in Fig. S2.

The resulting principal component (PC) scores plots for both the MTS multiplex (a) and the solution multiplex (b) are shown in Fig. 3. Both plots demonstrate a strong separation between the three single nanotags and the triplex allowing four distinct groupings to be observed. In both scores plots, the SESORRS spectra from the triplex is tightly clustered and clearly separated from the single nanotag samples. Thus, by using the scores plot from the solution multiplex (Fig. 3b), where the identity of the nanotags and the ratio of the nanotags in the instance of the triplex sample is known, it is possible to use the scores plot as a reference to develop a multiplex imaging system at depth using
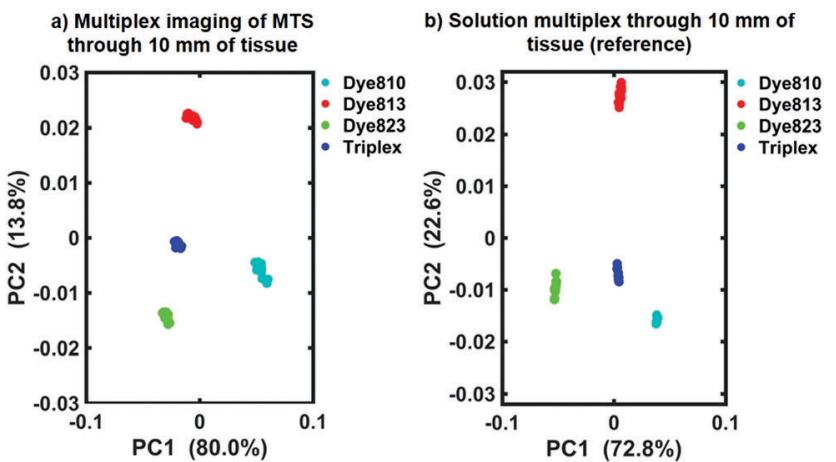

Fig. 3 PC scores plots discriminating between the single nanotags and the triplex of all three nanotags uptaken into MTS models (a) and in solution (b) through $10 \mathrm{~mm}$ of porcine tissue. In both PC plots, the red cluster refers to the MTS models or solution containing dye 813 , the teal cluster dye 810 and the green cluster dye 823 . Similarly, the blue cluster refers to either the MTS models or solution containing the triplex, i.e. equal contributions of dye 823 , dye 813 and dye 810 . Distinct separation is seen in both scores plots with the triplex falling in the middle of the three reference single nanotag clusters.

the SESORRS technique. The PC scores plot allows for not only the successful identification, but also discrimination between single and multiplexed SERRS nanotags taken up into tumour models, buried at depths of $10 \mathrm{~mm}$ using a handheld SORS spectrometer. The results presented here represent the ability to not only analyse a tissue sample containing SERRS-active nanotags at depth, but also the ability to simultaneously classify a sample containing multiple nanotags. The ability to distinguish between multiple SERS analytes has previously been reported 
using confocal techniques ${ }^{7}$ however the results presented here offer the added advantage of superior depth penetration through the use of backscattering SESORRS. To the best of our knowledge, this is the first report of the use of PCA in combination with SESORRS to spectrally discriminate between multiple nanotags taken up into ex vivo tumour models through depth. Previous work has reported the multiplexing potential of SESORS, ${ }^{8}$ however in that instance, the nanotags were located in different regions of a tissue section, thus a spatial multiplex was carried out. However the work presented here incorporates multiple nanotags into a MTS tumour model, thus representing a step forward in the ability to spectrally classify singleplex nanotags and a triplex of nanotags through $10 \mathrm{~mm}$ of tissue. Additionally, since the dyes are so spectrally similar, the potential of SESORRS combined with powerful data analysis to distinguish between multiple analytes through large tissue thicknesses is demonstrated.

Through utilising the powerful performance of chalcogenpyrylium-based Raman reporters for superior depth penetration, the ability to identify and discriminate between three different SERRS nanotags taken up into 3D breast cancer tumour models buried at depths of $10 \mathrm{~mm}$ is presented. To the best of our knowledge, this is the first report of the use of ex vivo breast cancer models for the development of a multiplex imaging system at depth using back-scattering SESORRS. In addition, by utilising PCA, the potential to classify multiple nanotags taken up into single tumour model using SESORRS is demonstrated for the first time. This is the first true multiplex using the SESORS technique since the approach classifies singleplex nanotags from a triplex of co-located nanotags, and is the largest thickness to which multiplex detection has been achieved through tissue using not only a handheld back-scattering approach but also the SESORRS technique. Previous work utilising a transmission geometry has shown the potential to multiplex through $20 \mathrm{~mm}$ of tissue. However, these results demonstrate the ability to multiplex through the same total thickness using handheld SESORRS with a back-scattering approach. The incident photons reach the MTS models at depths of $10 \mathrm{~mm}$ however, following interaction between the nanotags within the MTS models and the incident laser light, the scattered photons are then returned to the collection optics through $10 \mathrm{~mm}$ of tissue, thus the total thickness that the photons must travel through is equal to $20 \mathrm{~mm}$. Previous reports demonstrating SERS multiplexing using a transmission approach have involved benchtop systems which are capable of longer acquisition times and improved signal to noise, however the handheld instrument used in this instance is potentially more suited to clinical applications due to its portable and user-friendly nature. The ability to simultaneously detect multiple targets in vivo is a significant challenge, however through the exploitation of the resonance effect this novel work represents a significant step forward in the ability to not only detect but classify multiple vibrational fingerprints in vivo.
Future work will focus on expanding on this proof of concept study by detecting and imaging multiple targets in vivo using the SESORRS technique, as well as determining the minimum concentration of NPs required for detection through a given thickness.

FN acknowledge the help of Stuart Bonthron at Cobalt Light Systems. This work was supported by DSTL and the EPSRC [EP/J500550/1 and EP/M506643/1, KF and FN and EP/L014165/1, SM, DG and KF] and by the NSF [CHE-1566142, KP and MRD]. Research data associated with this paper will become available through the following link: http://dx.doi.org/10.15129/ee7384eb7d33-446d-aeb3-23003800bad8.

\section{Conflicts of interest}

The authors declare no conflict of interest.

\section{Notes and references}

1 The World Health Organisation, Cancer Fact Sheet, http://www.who. int/mediacentre/factsheets/fs297/en/, accessed 15 February 2018.

2 A. I. Henry, B. Sharma, M. F. Cardinal, D. Kurouski and R. P. Van Duyne, Anal. Chem., 2016, 88, 6638-6647.

3 U. S. Dinish, G. Balasundaram, Y. T. Chang and M. Olivo, Sci. Rep., 2014, 4, 4075.

4 X. Qian, X.-H. Peng, D. O. Ansari, Q. Yin-Goen, G. Z. Chen, D. M. Shin, L. Yang, A. N. Young, M. D. Wang and S. Nie, Nat. Biotechnol., 2008, 26, 83-90.

5 A. Oseledchyk, C. Andreou, M. A. Wall and M. F. Kircher, ACS Nano, 2017, 11, 1488.

6 S. Schlucker, Angew. Chem., Int. Ed., 2014, 53, 4756-4795.

7 C. L. Zavaleta, B. R. Smith, I. Walton, W. Doering, G. Davis, B. Shojaei, M. J. Natan and S. S. Gambhir, Proc. Natl. Acad. Sci. U. S. A., 2009, 106, 13511-13516.

8 N. Stone, M. Kerssens, G. R. Lloyd, K. Faulds, D. Graham and P. Matousek, Chem. Sci., 2011, 2, 776-780.

9 F. Nicolson, L. E. Jamieson, S. Mabbott, N. C. Shand, D. Graham and K. Faulds, J. Raman Spectrosc., 2017, 48, 1828-1838.

10 D. I. Ellis, R. Eccles, Y. Xu, J. Griffen, H. Muhamadali, P. Matousek, I. Goodall and R. Goodacre, Sci. Rep., 2017, 7, 12082.

11 K. Sowoidnich, J. H. Churchwell, K. Buckely, A. E. Goodship, A. W. Parker and P. Matousek, Analyst, 2017, 142, 3219.

12 N. Stone and P. Matousek, Cancer Res., 2008, 68, 4424-4430.

13 K. Ma, J. M. Yuen, N. C. Shah, J. T. Walsh, M. R. Glucksberg and R. P. Van Duyne, Anal. Chem., 2011, 83, 9146-9152.

14 A. S. Moody, P. C. Baghernejad, K. R. Webb and B. Sharma, Anal. Chem., 2017, 89, 5688-5692.

15 B. Sharma, K. Ma, M. R. Glucksberg and R. P. Van Duyne, J. Am. Chem. Soc., 2013, 135, 17290-17923.

16 N. Stone, K. Faulds, D. Graham and P. Matousek, Anal. Chem., 2010, 82, 3969-3973.

17 F. Nicolson, L. E. Jamieson, S. Mabbott, K. Plakas, N. Shand, M. Detty, D. Graham and K. Faulds, Chem. Sci., 2018, 9, 3788-3792.

18 M. A. Bedics, H. Kearns, J. M. Cox, S. Mabbott, F. Ali, N. C. Shand, K. Faulds, J. B. Benedict, D. Graham and M. R. Detty, Chem. Sci., 2015, 6, 2302-2306.

19 L. E. Jamieson, V. L. Camus, P. O. Bagnaninchi, K. M. Fisher, G. D. Stewart, W. H. Nailon, D. B. McLaren, D. J. Harrison and C. J. Campbell, Nanoscale, 2016, 8, 16710-16718.

20 H. Kearns, R. Goodacre, L. E. Jamieson, D. Graham and K. Faulds, Anal. Chem., 2017, 89, 12666-12673. 